\section{SEX DIFFERENCES IN THE TRANSCRIPTIONAL PROFILES OF MUCOSAL-ASSOCIATED INVARIANT T CELLS IN NEOADJUVANT ANTI-PD-1 TREATED NON-SMALL CELL LUNG CANCER (NSCLC)}

${ }^{1}$ Poromendro Burman*, 'Boyang Zhang, ${ }^{2}$ Zhicheng Ji, 'Justina Caushi, 'Frank Housseau, ${ }^{1}$ Andrew Pardoll, 'Zhang Jiajia, ${ }^{1}$ Hongkai Ji, 'Kellie Smith. 'Johns Hopkins School of Medicine, Baltimore, MD, USA; ${ }^{2}$ Duke University, Durham, NC, USA

Background Mucosal Associated Invariant T Cells (MAIT cells) are unconventional $\mathrm{T}$ cells that recognize vitamin $\mathrm{B}$ metabolites derived from bacteria and are mainly present in mucosal tissues and peripheral blood. ${ }^{1}$ Their activation by $\mathrm{T}$ Cell Receptor (TCR)-dependent and -independent pathways can result in effector function that can either promote or inhibit cytotoxic effects. ${ }^{2}$ MAIT cells are known to be involved in the pathogenesis of multiple diseases that involve mucosal tissues, such as non-small cell lung cancer (NSCLC). ${ }^{2}$ Recently, studies have shown that disparate outcomes to SARS-CoV-2-infection between males and females may involve a differential activation of MAIT cells in the lung mucosa. ${ }^{3}$ It is therefore conceivable to hypothesize that sex differences of MAIT cells in NSCLC may also impact outcome, however their involvement in progression and subsequent treatment response of NSCLC has never been explored.

Methods To study the transcriptional program of MAIT cells in NSCLC as a function of sex, peripheral blood and tissue biospecimens were obtained from the first-in-human clinical trial of neoadjuvant anti-PD-1 (nivolumab) in resectable nonsmall cell lung cancer; NCT02259621. ${ }^{4}$ Coupled single-cell RNAseq/TCRseq was performed on tumor infiltrating lymphocytes (TIL), paired adjacent normal lung, and tumor-draining lymph nodes (TDLN). MAIT cells were identified by expression of SLC4A10 and the invariant TRAV1-2 and TRAJ33/12/ 20 TCR. Computational analysis revealed 4 distinct MAIT cell clusters and differentially expressed genes in the tumors and healthy normal lung of males as compared to females.

Results In MAIT cells from females, we found upregulation of CD8A, GNLY, and NKG7 genes. These genes are involved with $\mathrm{T}$ cell activation and cytolytic function, suggesting that the activation of these genes in MAIT cells could be contributing towards their cytolytic activity in females. In MAIT cells from males, we found upregulation of PDE3B and PCBP2 genes, which are known to be involved with immunosuppression and downregulation of cytotoxic $\mathrm{T}$ lymphocyte (CTL) responses. These findings were consistent in the healthy normal lung, suggesting these transcriptional programs may be due to the normal lung biology and not necessarily a byproduct of carcinogenesis.

Conclusions These results highlight the potential for dual characteristics of MAIT cells in neoadjuvant anti-PD-1-treated NSCLCs and provide an important foundation in our study of the often dichotomous responses between males and females to immunotherapy. Future analyses will focus on the interplay of MAIT cells with other cells in the tumor microenvironment (TME) as a function of immunotherapy treatment and clinical response.

\section{REFERENCES}

1.. Chen Z, Wang H, D'Souza C, et al. Mucosal-associated invariant T-cell activation and accumulation after in vivo infection depends on microbial riboflavin synthesis and co-stimulatory signals. Mucosal Immunol 2017;10:58-68.

2.. Wen $X$, Zhang $X$, et al. Title of article: mucosal-associated invariant $T$ cells in lung cancers. Elsevier 2021;94.

3.. Yu C, Littleton S, et al. Mucosal-associated invariant T cell responses differ by sex in COVID-19. CellPress 2021;2:755-772.
4.. Caushi JX, Zhang J, Ji Z, et al. Transcriptional programs of neoantigen-specific TIL in anti-PD-1-treated lung cancers. Nature 2021.

Ethics Approval This study was approved by the Institutional Review Boards (IRB) at Johns Hopkins University (JHU) and Memorial Sloan Kettering Cancer Center and was conducted in accordance with the Declaration of Helsinki and the International Conference on Harmonization Good Clinical Practice guidelines. The patients described in this study provided written informed consent.

http://dx.doi.org/10.1136/jitc-2021-SITC2021.286 